\title{
Domestic Wastewater Reclamation Coupled with Biofuel/Biomass Production Based on Microalgae: A Novel Wastewater Treatment Process in the Future
}

\author{
Hong-Ying HU***, Xin LI*, Yin YU*, Yin-Hu WU*, Masaki SAGEHASHI***, Akiyoshi \\ SAKODA**** \\ *Environmental Simulation and Pollution Control State Key Joint Laboratory, School of \\ Environment, Tsinghua University, Beijing 100084, P. R. China \\ **Graduate School at Shenzhen, Tsinghua University, Shenzhen 518055, P. R. China \\ ***Center of Education for Leaders in Environmental Sectors, Tokyo University of Agriculture \\ and Technology, Tokyo 184-8588, Japan \\ ****Institute of Industrial Science, The University of Tokyo, Tokyo 153-8505, Japan
}

\begin{abstract}
Freshwater shortage and energy crisis are the two major challenges in the $21^{\text {st }}$ century. The coupling of wastewater treatment and energy production to supply reclaimed water and sustainable energy is a very promising approach to handle these two challenges. A novel process of domestic wastewater reclamation coupled with biofuel/biomass production based on microalgae is proposed in this paper. The organic pollutants in wastewater are separated and concentrated by flocculation and filtration, and nitrogen and phosphorus are used as nutrients for microalgal cultivation. The organics separated and microalgal biomass harvested can be further processed to produce biofuel. The novel process proposed in this paper will bring about innovation in wastewater treatment, and the conventional wastewater treatment process will be transformed from a mere "treatment process" to a "production process" using wastes as raw materials and thereby can produce biofuel and other valuable by-products. At present, this novel process is in the conceptual stage, and some key techniques necessary to be further studied are also discussed in this paper.
\end{abstract}

Keywords: biomass production, microalgae-based biofuel, organic pollutants separation, wastewater treatment, water reuse

\section{INTRODUCTION}

The global population expansion and the quick development of economy and industry generate large amount of wastewater, and also require plenty of freshwater. However, freshwater in our planet is limited and scarce, especially when the need for freshwater increases extensively.

Facing the challenge of freshwater shortage, water reuse is the only possible way to ease the pressure of the present situation. The key point in water reuse is the guaranteed quality of reclaimed water according to different pathways of reuse. For example, for landscape reuse, the concentration of nitrogen and phosphorus in the reclaimed water must be reduced to certain level in order to avoid eutrophication and deterioration in the landscape water quality (Li et al., 2009; Li et al., 2010b).

Although there is an urgent demand for the proper treatment of the increasing amount of wastewater for water reuse, limitations exist in the present wastewater treatment and reclamation processes:

\footnotetext{
Address correspondence to Hong-Ying HU, School of Environment, Tsinghua University, Email: hyhu@tsinghua.edu.cn

Received September 12, 2010, Accepted November 20, 2010.
} 
(1) The present wastewater treatment processes are really energy-consuming. Large amount of energy is necessary for pump operation, aeration, sludge disposal and other steps involved in the process. Due to the present energy crisis, energy-consuming treatment processes without any valuable by-products or energy produced do not promote a sustainable future.

(2) In the aerobic tank for the treatment of organic pollutants, $\mathrm{CO}_{2}$ is released due to the microorganisms' respiration, which would aggravate the greenhouse effect and contribute to global warming.

(3) The removal efficiency for nitrogen and phosphorus is relatively low by biological treatment, especially for nitrogen (Vílchez et al., 1997). Chemical precipitation has an obvious effect on phosphorus removal, but the cost of chemical reagents is high and the excess sludge is difficult to deal with (De-Bashan and Bashan, 2004).

(4) While the conventional treatment process removes organic pollutants in wastewater, large amount of excess sludge is generated during the assimilation of organics by microorganisms in the activated sludge. Therefore, the wastes are transferred from liquid phase to solid phase and the disposal of sludge (e.g. dewatering, stabilization and other procedures) is also highly energy-consuming.

Besides the freshwater shortage problem, another challenge faced by the people in the $21^{\text {st }}$ century is the energy crisis. Fossil fuel is not a renewable energy source, and is going to be scarce due to the rapid development of the society and the excessive consumption of energy (Vasudevan and Briggs, 2008). Meanwhile, combustion of fossil fuel would release huge amount of $\mathrm{CO}_{2}$. Thus, the search for a sustainable and environment-friendly energy source is the major approach to handle the challenge of energy crisis. Microalgae-based biodiesel is a very attractive energy source and is a high potential fossil fuel substitute, because of microalgae's fast growth rate and high lipid content (Bruce, 2008; Groom et al., 2008; Li et al., 2010c). However, the high cost of microalgae-based biodiesel is the main bottleneck of its commercial application, and the cultivation cost (mainly referring to growth medium such as fresh water and inorganic nutrients) represents over $70 \%$ of the total cost (Behzadi and Farid, 2007). Therefore, reducing the microalgal cultivation cost is greatly necessary in this research.

Coupling of wastewater treatment and the production of microalgae-based biodiesel/biomass is an effective way to enhance the inorganic nutrient removal by microalgae and to reduce the cost of the growth medium for microalgal cultivation $(\mathrm{Hu}$ et al., 2009; Hu et al., 2010; Li et al., 2010a). Based on this concept, a novel process of domestic wastewater reclamation coupled with biofuel/biomass production based on microalgae is proposed in this paper.

\section{CONCEPT DESIGN}

\section{Design and description of the novel process}

A novel process being proposed in this study for domestic wastewater reclamation coupled with biofuel/biomass production based on microalgae is shown in Fig. 1. The primary settlement tank in the novel process is the same as the conventional one. 
However, a flocculation and filtration system subsequently replaces the conventional aerobic tank. Compared to the conventional treatment process, the organic pollutants in this novel process are no longer degraded to $\mathrm{CO}_{2}$ by the microorganisms in activated sludge process, but are concentrated and separated by flocculation and filtration with organic flocculants which may be made from the wetland plants as will be discussed later. In this process, high-concentration of organics can be acquired through flocculation and filtration, and therefore, water with low concentration of organic pollutants is obtained after the filtration process.

In the novel process, nitrogen and phosphorus would not be removed through $\mathrm{A}^{2} \mathrm{O}$ method (anaerobic-anoxic-oxic process) as the conventional one does. The inorganic nutrients in wastewater are not even considered as pollutants anymore, instead, they are seen as a kind of resource which could be used to cultivate microalgae. The microalgal cultivation system could be an open pond (e.g. raceway pond, high-rate algal pond, etc.) or a closed photobioreactor, according to the practical conditions in wastewater treatment plant. The technique of carbon capture and storage (CCS) could be applied here to supply $\mathrm{CO}_{2}$ to microalgal cultivation. The $\mathrm{CO}_{2}$ gas could be the waste gas from the power plant or some other places, so this is also an approach of $\mathrm{CO}_{2}$ fixation. In the microalgal cultivation system the microalgae grow with inorganic nutrients in wastewater as growth medium, and at the same time the nitrogen and phosphorus in wastewater are removed efficiently.

After the microalgal cultivation system, the microalgal cells need to be separated and harvested from the wastewater. According to the property of microalgal cells cultivated, different harvest methods, such as flocculation, flotation, centrifugation, and filtration, could be applied. By separating microalgal cells from the wastewater, clean water with low content of organics and inorganic nutrients could be obtained, and also the microalgal biomass could be harvested for further application. The clean water from the microalgae-separation unit could be reused in the environment and landscape through artificial wetland for further removal of inorganic nutrients and other pollutants, or be reused in municipal services and industries. Meanwhile, the plants harvested from the artificial wetland could be processed to produce valuable by-products, such as organic flocculants which could be used in the flocculation unit in the former treatment process of flocculation and filtration.

The microalgal biomass harvested from the microalgal cultivation system could be used as the feedstock of biofuel. The lipid could be extracted from the microalgal biomass and produce biodiesel through transesterification. The oil cakes (residual microalgal cells after lipid extraction), microalgal cells from microalgae-separation unit, high-concentration organics from the flocculation and filtration unit, and the primary settled sludge from the primary settlement tank could be treated by anaerobic fermentation to produce biogas (mainly $\mathrm{CH}_{4}$ ), and the residuals from fermentation could be used as organic fertilizers.

Therefore, from the novel process we can see that, the inputs of the coupled system are wastes (wastewater, waste $\mathrm{CO}_{2}$ ), and the outputs are valuable products (reclaimed water, biofuel/biomass, organic fertilizer and organic flocculants). 


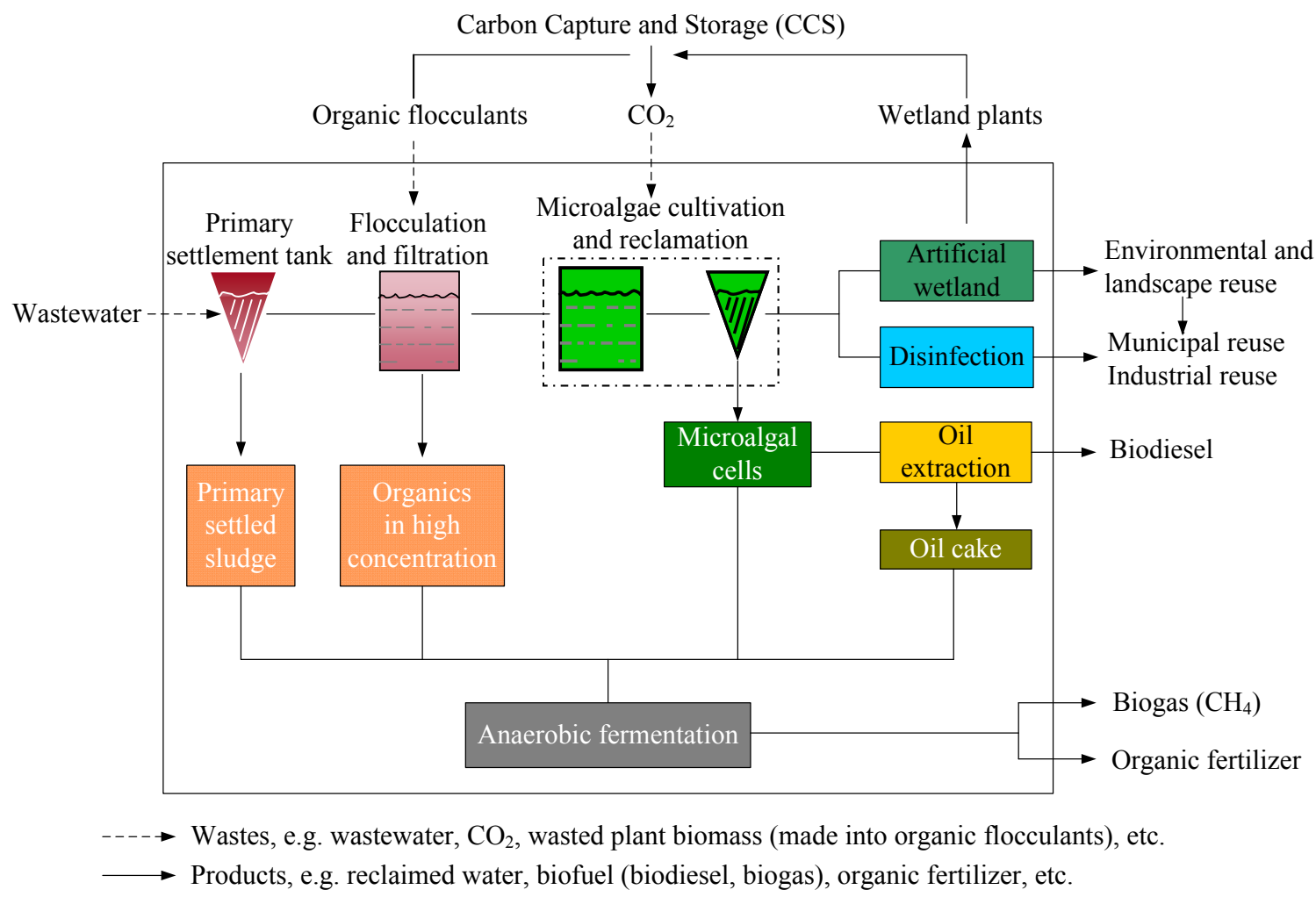

Fig. 1 - Schematic diagram of the novel process of domestic wastewater reclamation coupled with biofuel/biomass production based on microalgae

\section{Novel concepts}

Several novel concepts for the novel process proposed for domestic wastewater reclamation coupled with biofuel/biomass production based on microalgae are as follows:

(1) Organic pollutants are no longer degraded to $\mathrm{CO}_{2}$ and transferred to sludge by activated sludge process. Instead, organic pollutants are collected through flocculation and filtration by organic flocculants for further anaerobic fermentation to produce biogas.

(2) The $\mathrm{A}^{2} \mathrm{O}$ process is omitted, and the inorganic nutrients are used to cultivate microalgae, which can be further processed to obtain biodiesel and other biofuels.

(3) With CCS technique and microalgal cultivation, the novel process could be seen as a sink of $\mathrm{CO}_{2}$ and offers an effective way for $\mathrm{CO}_{2}$ fixation and utilization.

(4) Wastewater is not considered as waste anymore, but is used as a kind of resource to produce biofuel and other valuable by-products. Therefore, the wastewater treatment process is transformed from being a mere "treatment process" into a "production process". 


\section{ESTABLISHMENT OF THE NOVEL PROCESS \\ Key techniques needed for the novel process}

At the moment, the novel process is just a concept, and there is limited research on its key techniques for both the wastewater treatment and biofuel/biomass production. In the novel process, the primary settlement tank units, anaerobic fermentation, artificial wetland and disinfection all have relatively established techniques that exist in the present wastewater treatment plants. However, the flocculation and filtration of organic pollutants by organic flocculants made from plant biomass, the microalgae cultivation unit and the downstream process to produce biofuel are the key points of the novel process, which determine the wastewater treatment efficiency and the net energy production of the whole process. Therefore, the key techniques of the novel process are indentified and listed below.

\section{Flocculation of organic pollutants by organic flocculants}

Inorganic flocculants, such as aluminum and ferric salts, are popular at present. However, these inorganic flocculants are not suitable to be used in the novel process for the flocculation of the organic pollutants in wastewater, because the flocculated and filtrated organics need to be further fermented anaerobically and the inorganic flocculants contained in the filtrated organics could not be processed in the anaerobic fermentation. Therefore, efficient organic flocculants need to be utilized. In addition, it would be great if the organic flocculants could be made from the plant biomass in the artificial wetland, which could form a closed production chain in the novel process. To guarantee the effluent quality, there are still lots of research work to be done such as utilization, evaluation and field tests for the practical application of the organic flocculants.

\section{Selection of microalgal species suitable for the coupled system}

In the novel process being proposed, the proper selection of microalgal species is the foundation and key point of the microalgal cultivation unit. Considering the inorganic nutrient removal and the biofuel/biomass production, the selection rules should contain: high growth rate in secondary effluent of the wastewater; high removal efficiency of nitrogen and phosphorus; and the accumulation of high lipid content in microalgal cells (Li et al., 2010d).

Many researches have reported the oily microalgal species with high lipid content, such as Chlorella vuglaris, Botryococcus braunii, and Haematococcus pluvialis among others (Illman et al., 2000; Chisti, 2008). However, these oily microalgal species may not be adaptable to the secondary effluent of wastewater. Li et al (2010d) isolated a freshwater microalga Scenedesmus sp. LX1 with high lipid content (around 25 - 35\%) from low-nutrient environment, and they compared Scenedesmus sp. LX1 with other reported 11 oily microalgal species based on the growth and lipid accumulation properties while growing in the secondary effluent of domestic wastewater, and the results are shown in Fig. 2. Most of the oily microalgal species reported in literature could not grow in the secondary effluent of domestic wastewater, but Scenedesmus sp. LX1 grew best and accumulated the highest lipid content in microalgal cells.

Algae isolated from wastewater treatment plant sites or real water bodies can usually adapt to culture conditions and grow better (Pérez et al., 2004). Therefore, proper 

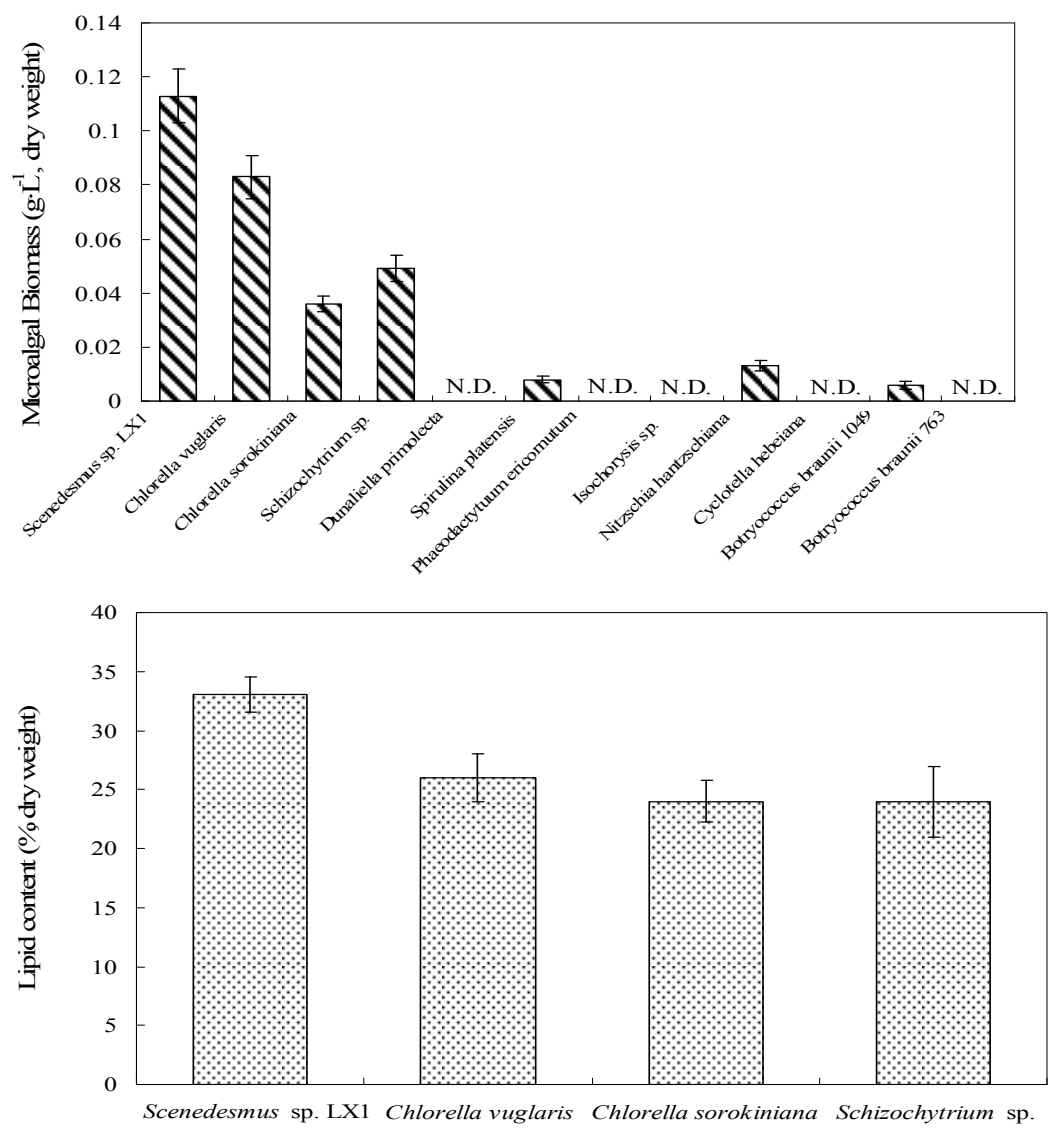

Fig. 2 - Comparison of Scenedesmus sp. LX1 and other 11 oily microalgal species based on the growth and lipid accumulation properties in the secondary effluent of a domestic wastewater (Li et al., 2010d)

selection and isolation of microalgal species are very important in the microalgal cultivation unit of the novel process.

\section{Harvest of microalgal biomass}

The microalgal biomass needs to be separated and harvested from the microalgal cultivation unit for further processing and production of biofuel. The typical microalgal biomass is in the range of $2-3 \mathrm{~g} / \mathrm{L}$ (dry weight) in general cultivation process, and under some special conditions the microalgal biomass could reach $5 \mathrm{~g} / \mathrm{L}$ (dry weight). The microalgal biomass which satisfies the industrial process demand should be in the range of $300-400 \mathrm{~g} / \mathrm{L}$ (dry weight), so the algal culture needs to be concentrated by 100 - 1000 fold in order to be used as feedstock for biofuel production.

The harvest and concentration of microalgal biomass are highly energy-consuming and considered to be the second largest cost in microalgal biodiesel production (Wang et al., 2008). Thus, it is essential to choose a suitable harvest and concentration technology in the novel process. The common harvesting techniques for microalgal biomass include natural sedimentation (Grenn et al., 1996), flocculation (Munoz and Guieysse, 2006), flotation (Grenn et al., 1996), mobilization (Olguín, 2003), centrifugation (Chisti, 2007), filtration and membrane separation. Among all these techniques, membrane separation 


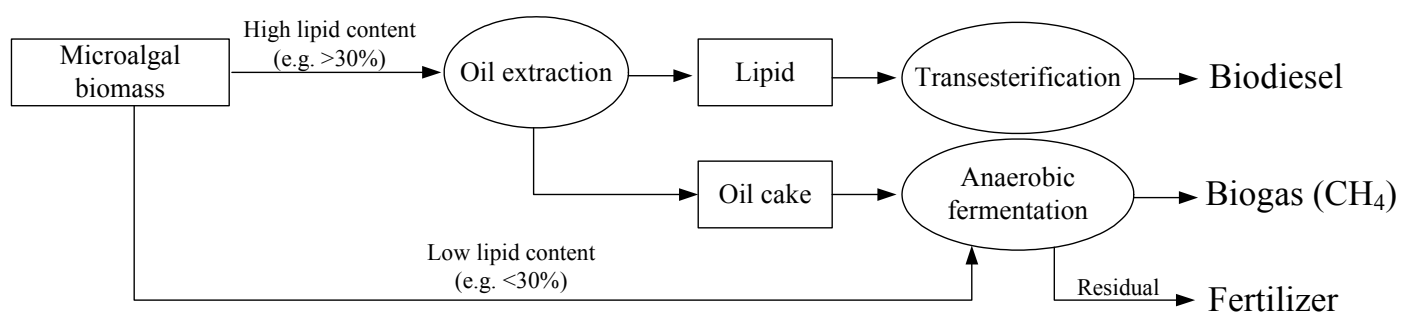

Fig. 3 - Schematic diagram of the thorough utilization of microalgal biomass to produce biofuel

is technologically developed and has a very good separation effect.

\section{Thorough utilization of microalgal biomass}

After lipid extraction, the disposal and utilization of oil cakes (residual microalgal cells) is another important problem. On one hand, nitrogen and phosphorus are still contained in the oil cakes, so improper disposal of the oil cakes would have negative effects on the environmental quality (Bruno et al., 2009). On the other hand, the protein, hydrocarbon and other organic materials that existed in oil cakes still store large amount of energy which may exceed the one contained in biodiesel produced by microalgal lipid (Lardon et al., 2009), so it is meaningful to recover the energy contained in oil cakes to get the maximum net energy production.

The suggested thorough utilization of microalgal biomass to produce the maximum biofuel and by-products is shown in Fig. 3. While the microalgal biomass has high lipid content (e.g. $>30 \%$ ), the lipid can be extracted and then processed to produce biodiesel through transesterification, and the oil cake can be sent to the anaerobic fermentation unit to produce biogas $\left(\mathrm{CH}_{4}\right)$. However, if the microalgal biomass has relatively low lipid content (e.g. $<30 \%$ ), then it would be more energy-efficient to send the whole microalgal biomass to anaerobic fermentation to produce biogas. The residual materials after anaerobic fermentation could be used as organic fertilizer in farmlands.

\section{CONCLUSION}

In this study, the novel process being proposed for domestic wastewater reclamation coupled with biofuel/biomass production based on microalgae considers wastewater as a kind of resource instead of just waste, and shifts the wastewater treatment process from being a mere "treatment process" into a "production process". While treating wastewater, it can produce biofuel and other valuable by-products as well as fix $\mathrm{CO}_{2}$. Although at the moment the novel process is just a concept and still leaves several key techniques to be studied, under the severe challenges of freshwater shortage and energy crisis, this novel process of domestic wastewater would have a promising future.

\section{ACKNOWLEDGEMENT}

This study was supported by China National Science Fund for Distinguished Young Scholars (No.50825801), and National Natural Science Foundation of China (NSFC) Japan Science and Technology Agency (JST) Joint Program (Strategic International Cooperative Program). 


\section{REFERENCES}

Behzadi S. and Farid M. M. (2007). Review: examining the use of different feedstock for the production of biodiesel, Asia-Pac. J. Chem. Eng., 2, 480-486.

Bruno S., Nicolas B. and Olivier B. (2009) Anaerobic digestion of microalgae as a necessary step to make microalgal biodiesel sustainable, Biotechnol. Adv., 27, 409-416.

Bruce E. R. (2008). Opportunities for renewable bioenergy using microorganisms, Biotechnol. Bioeng., 100(2), 203-212.

Chisti Y. (2007). Biodiesel from microalgae, Biotechnol. Adv., 25, 294-306.

Chisti Y. (2008). Biodiesel from microalgae beats bioethanol, Trends Biotechnol., 26(3), 126-131.

De-Bashan L. E. and Bashan Y. (2004). Recent advances in removing phosphorus from wastewater and its future use as fertilizer (1997-2003), Water Res., 38, 4222-4246.

Grenn F., Bernstone L. and Lundquist T. (1996). Advanced integrated wastewater pond systems for nitrogen removal, Wat. Sci. and Tech., 33(7), 207-217.

Groom M. J., Gray E. M. and Townsend P. A. (2008). Biofuels and biodiversity: principles for creating better policies for biofuel production, Conserv. Biol., 22(3), 602-609.

$\mathrm{Hu}$ H. Y. and Li X. (2010). Analysis of key techniques and production potential of biodiesel production based on microalgae with wastewater as resources, Eco. and Env. Sci., 19(3), 739-744. (in Chinese)

Hu H. Y., Li X. and Yang J. (2009). Coupling of wastewater deep purification and high quality biomass production based on microalgae cultivation, Eco. and Env. Sci., 18(3), 1122-1127. (in Chinese)

Illman A. M., Scragg A. H. and Shales S. W. (2000). Increase in Chlorella strains calorific values when grown in low nitrogen medium, Enzyme Microb. Technol., 27, 631-635.

Lardon L., Hélias A., Sialve B., Steyer J. P. and Bernard O. (2009). Life-cycle assessment of biodiesel production from microalgae, Environ. Sci. Technol., 43(17), 6475-6481.

Li X., Hu H. Y., Gan K. and Sun Y. X. (2010a). Effects of different nitrogen and phosphorus concentrations on the growth, nutrient uptake, and lipid accumulation of a freshwater microalga Scenedesmus sp, Bioresour. Technol., 101, 5494-5500.

Li X., Hu H. Y., Gan K. and Yang J. (2010b). Growth and nutrient removal properties of a freshwater microalga Scenedesmus sp. LX1 under different kinds of nitrogen sources, Ecol. Eng., 36, 379-381.

Li X., Hu H. Y. and Yang J. (2010c). Effect of LED's red/blue light on the growth characteristic and lipid production of Scenedesmus sp. LX1, Env. Sci., 31(2), 244-250. (in Chinese)

Li X., Hu H. Y. and Yang J. (2010d). Lipid accumulation and nutrients removal properties in secondary effluent of a newly-isolated freshwater microalga Scenedesmus sp. LX1, New Biotechnol., 27(1), 59-63.

Li X., Hu H. Y., Yang J. and Bai Y. (2009). Method to establish the nitrogen and phosphorus standard of reclaimed water reused in landscape, Eco. and Env. Sci., 18(6), 2404-2408. (in Chinese)

Munoz R. and Guieysse B. (2006). Algal-bacterial processes for the treatment of hazardous contaminants: A review, Water Res., 40, 2799-2815. 
Olguín, E. (2003). Phycoremediation: key issues for cost-effective nutrient removal processes, Biotechnol. Adv., 22, 81-91.

Pérez M. V. J., Castillo P. S., Romera O., Moreno D. F. and Martínez C. P. (2004). Growth and nutrient removal in free and immobilized planktonic green algae isolated from pig manure, Enzyme Microb. Technol., 34, 392-398.

Vasudevan P. T. and Briggs M. (2008). Biodiesel production-current state of the art and challenges, J. Ind. Microbiol. Biotechnol., 35, 421-430.

Vílchez C., Garbayo I. and Lobato M. V. (1997). Microalgae-mediated chemicals production and wastes removal, Enzyme Microb. Technol., 20, 562-572.

Wang B., Li Y., Wu N. and Lan C. Q. (2008). $\mathrm{CO}_{2}$ bio-mitigation using microalgae, Appl. Microbiol. Biot., 79, 707-718. 